Confluentes Mathematici, Vol. 1, No. 1 (2009) 87-109

(c) World Scientific Publishing Company

\title{
DETECTING INTEGRAL POLYHEDRAL FUNCTIONS
}

\author{
KIRAN S. KEDLAYA* and PHILIP TYNAN ${ }^{\dagger}$ \\ Department of Mathematics, \\ Massachusetts Institute of Technology, \\ 77 Massachusetts Avenue \\ Cambridge, MA 02139-4307, USA \\ *kedlaya@mit.edu \\ †ptynan@mit.edu \\ Received 26 September 2008 \\ Revised 29 January 2009
}

\begin{abstract}
We study the class of real-valued functions on convex subsets of $\mathbb{R}^{n}$ which are computed by the maximum of finitely many affine functionals with integer slopes. We prove several results to the effect that this property of a function can be detected by sampling on small subsets of the domain. In so doing, we recover in a unified way some prior results of the first author (some joint with Liang Xiao). We also prove that a function on $\mathbb{R}^{2}$ is a tropical polynomial if and only if its restriction to each translate of a generic tropical line is a tropical polynomial.
\end{abstract}

Keywords: Convex functions; integral polyhedral functions; tropical polynomials.

\section{Introduction}

One of the most fundamental classes of real-valued functions on $\mathbb{R}^{n}$ is the class of convex polyhedral functions, i.e. those functions computed as the maximum of a finite number of affine functionals

$$
\lambda\left(x_{1}, \ldots, x_{n}\right)=a_{1} x_{1}+\cdots+a_{n} x_{n}+b .
$$

For one, this class is fundamental in the theory of linear programming; on the other hand, it also figures prominently in algebraic geometry via the study of tropical polynomial functions.

The purpose of this paper is to study some subclasses of convex polyhedral functions for which we impose some integrality conditions. The two classes we focus on are the transintegral polyhedral functions, for which the coefficients $a_{1}, \ldots, a_{n}$ in the affine functionals $\lambda$ must be integers, and the integral polyhedral functions, for which both the coefficients $a_{1}, \ldots, a_{n}$ and the constant term $b$ must be integers.

What we prove are a number of results of the following form: a function on a suitable convex subset of $\mathbb{R}^{n}$ is (trans)integral polyhedral if and only if the same is true of its restrictions to some small subsets of the domain (usually certain straight lines). In so doing, we recover in a unified way two earlier results along 
these lines, one (Theorem 8.2) from the solo paper [3] by the first author, the other (Theorem 7.4) from the joint paper [6] by the first author and Liang Xiao. (Those results were introduced to study $p$-adic differential equations; we include a brief description of that application.) We also obtain a theorem (Theorem 9.1) that asserts that a tropical Laurent polynomial may be identified from its restrictions to the translates of a generic tropical line.

\section{Convex Sets and Functions}

Notation 1.1. Throughout this paper, let $e_{1}, \ldots, e_{n}$ denote the standard basis vectors of $\mathbb{R}^{n}$.

Notation 1.2. For all definitions in this section and the next, fix a subfield $F$ of $\mathbb{R}$. When one of these definitions is referenced with $F$ omitted, one should take $F=\mathbb{R}$; the only other case we will be interested in is $F=\mathbb{Q}$.

Definition 1.3. Let $S$ be a subset of $F^{n}$ for some non-negative integer $n$. We say $S$ is $F$-convex (resp. $F$-affine) if for any $x, y \in S$ and $t \in[0,1] \cap F$ (resp. $t \in F$ ), we have $t x+(1-t) y \in S$. Any intersection of $F$-convex (resp. $F$-affine) sets is again $F$-convex (resp. $F$-affine).

Definition 1.4. For any set $T \subseteq F^{n}$, the $F$-convex hull (resp. F-affine hull) of $T$ is the intersection of all $F$-convex (resp. $F$-affine) sets of $F^{n}$ containing $T$. It is equal to the set of all points of the form $t_{1} x_{1}+\cdots+t_{m} x_{m}$ for some positive integer $m$, some $x_{1}, \ldots, x_{m} \in S$, and some $t_{1}, \ldots, t_{m} \in F \cap[0,+\infty)\left(\operatorname{resp} . t_{1}, \ldots, t_{m} \in F\right)$ with $t_{1}+\cdots+t_{m}=1$ [7, Corollary 1.4.1, Theorem 2.3].

Definition 1.5. For $S \subseteq \mathbb{R}^{n}$ convex, the (relative) interior of $S$, $\operatorname{denoted} \operatorname{int}(S)$, is defined to be the topological interior of $S$ relative to its affine hull. This is nonempty if $S$ is nonempty [7, Theorem 6.2]. By the dimension of $S$, denoted $\operatorname{dim}(S)$, we will mean the dimension of its affine hull.

Definition 1.6. Let $S \subseteq F^{n}$ be an $F$-convex set. A function $f: S \rightarrow \mathbb{R}$ is $F$-convex if for any $x, y \in S$ and $t \in[0,1] \cap F$, we have the Jensen inequality

$$
t f(x)+(1-t) f(y) \geq f(t x+(1-t) y) .
$$

This implies that for any $x_{1}, \ldots, x_{m} \in S$ and $t_{1}, \ldots, t_{m} \in[0,1] \cap F$ with $t_{1}+\cdots+$ $t_{m}=1$, we have

$$
\sum_{i=1}^{m} t_{i} f\left(x_{i}\right) \geq f\left(\sum_{i=1}^{m} t_{i} x_{i}\right) .
$$

If $f$ is convex, then $f$ is continuous on $\operatorname{int}(S)$ [7, Theorem 10.1]; we will prove a stronger result later (Theorem 5.1).

Remark 1.7. Note that $f$ is convex if and only if the epigraph of $f$, defined by

$$
\operatorname{epi}(f)=\{(x, y) \in S \times \mathbb{R}: y \geq f(x)\},
$$


is convex. Using this convention, we may extend the definition of convexity to functions with range $\mathbb{R} \cup\{ \pm \infty\}$ (but only when explicitly specified). In particular, one can canonically extend any convex function on $S$ to a convex function on $\mathbb{R}^{n}$ taking the value $+\infty$ everywhere on $\mathbb{R}^{n} \backslash S$; this is the convention used in [7].

\section{Directional Derivatives}

Definition 2.1. Let $S \subseteq F^{n}$ be an $F$-convex subset. Pick $x \in S$ and $z \in F^{n}$ such that $x+t z \in S$ for some $t \in(0,+\infty) \cap F$. For $f: S \rightarrow \mathbb{R}$ a function whose restriction to $\{x+t z: t \in[0, \epsilon] \cap F\}$ is $F$-convex for some $\epsilon>0$, define $f^{\prime}(x, z)$ to be the directional derivative of $f$ at $x$ in the direction of $z$, i.e.

$$
f^{\prime}(x, z)=\lim _{t \rightarrow 0^{+}} \frac{f(x+t z)-f(x)}{t} .
$$

Note that this is a limit taken over a decreasing sequence; for it to exist in all cases, we must allow it to take the value $-\infty$.

Lemma 2.2. Let $S \subseteq F^{n}$ be an $F$-convex subset. Let $U \subseteq S$ be an $F$-convex subset. Suppose $z \in F^{n}$ is such that for each $x \in U$, there exists $t \in(0,+\infty) \cap F$ such that $x+t z \in S$. Let $f: S \rightarrow \mathbb{R}$ be a function satisfying the following conditions:

(a) The restriction of $f$ to $U$ is affine.

(b) For each $x \in U$, there exists $\epsilon>0$ such that the restriction of $f$ to $\{x+t z: t \in$ $[0, \epsilon] \cap F\}$ is $F$-convex.

(c) For each line segment $L$ with endpoints in $U$, there exists $\epsilon>0$ such that for each $t \in[0, \epsilon] \cap F$, the restriction of $f$ to $\left(L \cap F^{n}\right)+t z$ is $F$-convex.

Then the function $x \mapsto f^{\prime}(x, z)$ is $F$-convex on $U$.

Proof. Take $x_{1}, x_{2} \in U$. We assume first that $f^{\prime}\left(x_{1}, z\right), f^{\prime}\left(x_{2}, z\right)>-\infty$. Pick $t \in[0,1] \cap F$ and put $x_{3}=t x_{1}+(1-t) x_{2}$. Choose $\epsilon>0$ satisfying (b) for each of $x=x_{1}, x_{2}, x_{3}$, and satisfying (c) for $L$ the line segment from $x_{1}$ to $x_{2}$. Pick $u \in(0, \epsilon] \cap F$ such that

$$
\frac{f\left(x_{i}+u z\right)-f\left(x_{i}\right)}{u} \leq f^{\prime}\left(x_{i}, z\right)+\delta, \quad i=1,2 .
$$

Then

$$
\begin{aligned}
t f^{\prime}\left(x_{1}, z\right)+(1-t) f^{\prime}\left(x_{2}, z\right) & \geq t \frac{f\left(x_{1}+u z\right)-f\left(x_{1}\right)}{u}+(1-t) \frac{f\left(x_{2}+u z\right)-f\left(x_{2}\right)}{u}-\delta \\
& =\frac{t f\left(x_{1}+u z\right)+(1-t) f\left(x_{2}+u z\right)-f\left(x_{3}\right)}{u}-\delta \\
& \geq \frac{f\left(x_{3}+u z\right)-f\left(x_{3}\right)}{u}-\delta \\
& \geq f^{\prime}\left(x_{3}, z\right)-\delta .
\end{aligned}
$$


Since $\delta$ was arbitrary, this proves the claim when both $f^{\prime}\left(x_{1}, z\right)$ and $f^{\prime}\left(x_{2}, z\right)$ are not $-\infty$. If one of them is $-\infty$, the same argument would imply that $f^{\prime}\left(x_{3}, z\right)=-\infty$; this completes the proof.

\section{Affine Functionals}

Notation 3.1. For all definitions in this section and the next, fix a subgroup $G$ of $\mathbb{R}$.

Definition 3.2. An affine functional is a map $\lambda: \mathbb{R}^{n} \rightarrow \mathbb{R}$ of the form $\lambda\left(x_{1}, \ldots, x_{n}\right)=a_{1} x_{1}+\cdots+a_{n} x_{n}+b$ for some $a_{1}, \ldots, a_{n}, b \in \mathbb{R}$. The slope of $\lambda$ is the linear functional $\mu$ defined by $\mu\left(x_{1}, \ldots, x_{n}\right)=a_{1} x_{1}+\cdots+a_{n} x_{n}$. We say $\lambda$ is $G$-integral if $a_{1}, \ldots, a_{n} \in \mathbb{Z}$ and $b \in G$. We use integral and transintegral as synonyms for $\mathbb{Z}$-integral and $\mathbb{R}$-integral, respectively.

We can characterize convexity using affine functionals as follows.

Lemma 3.3. Let $S \subseteq \mathbb{R}^{n}$ be an open convex subset. Consider the following conditions on a function $f: S \rightarrow \mathbb{R}$.

(a) $f$ is convex.

(b) For each $x \in S$, there exists an affine functional $\lambda: \mathbb{R}^{n} \rightarrow \mathbb{R}$ such that

$$
\begin{aligned}
& f(x)=\lambda(x) \\
& f(y) \geq \lambda(y) \quad(y \in S) .
\end{aligned}
$$

(c) For each $x \in S$, there exist an affine functional $\lambda$ and a quantity $\epsilon>0$ such that

$$
\begin{aligned}
& f(x)=\lambda(x) \\
& f(y) \geq \lambda(y) \quad(y \in S,|x-y|<\epsilon) .
\end{aligned}
$$

Then (a) and (b) are equivalent. Moreover, if $f$ is assumed to be upper semicontinuous, then (a) and (b) are also equivalent to (c).

Proof. If (a) holds, then (b) holds because $(x, f(x)) \notin \operatorname{int}(\operatorname{epi}(f))$, so by $[7$, Corollary 11.6.2] there is a linear function on $\mathbb{R}^{n+1}$ which is zero at $(x, f(x))$ and non-negative on epi $(f)$. If (b) holds, then (a) holds because epi $(f)$ is the intersection of the epigraphs of some affine functionals, and so is convex.

Clearly (b) implies (c). Conversely, suppose $f$ is upper semicontinuous and (c) holds. As noted in [8, Theorem 3], the convexity of $f$ in this case follows from the analogous statement with $n=1$, which in turn follows at once from $[1, \S 1.4$, Proposition 9, Corollaire 1].

Corollary 3.4. Let $S \subseteq \mathbb{R}^{n}$ be an open convex subset, let $f: S \rightarrow \mathbb{R}$ be a convex function, and let $T$ be the set of affine functionals $\lambda: \mathbb{R}^{n} \rightarrow \mathbb{R}$ such that $f(x) \geq \lambda(x)$ for all $x \in S$. Then for all $x \in S$,

$$
f(x)=\sup \{\lambda(x): \lambda \in T\} .
$$


Proof. The inequality $f(x) \geq \sup \{\lambda(x): \lambda \in T\}$ is evident from the definition of $T$. For the reverse inequality, note that for each $x \in S$, by Lemma 3.3, there exists $\lambda \in T$ with $f(x)=\lambda(x)$.

Corollary 3.5. With notation as in Corollary 3.4, the formula (3.4.1) defines a lower semicontinuous extension of $f$ to a convex function $\tilde{f}: \mathbb{R}^{n} \rightarrow \mathbb{R} \cup\{+\infty\}$.

Definition 3.6. For $S \subseteq \mathbb{R}^{n}$ a convex subset and $f: S \rightarrow \mathbb{R}$ a convex function, a domain of affinity for $f$ is a subset $U \subseteq S$ with nonempty interior relative to the affine hull of $S$, on which $f$ agrees with an affine functional $\lambda$. We call $\lambda$ an ambient functional for $U$; it is uniquely determined if $S$ has affine hull $\mathbb{R}^{n}$.

Lemma 3.7. Let $S \subseteq \mathbb{R}^{n}$ be a convex subset, let $f: S \rightarrow \mathbb{R}$ be a convex function, and let $\lambda$ be an ambient functional for some domain of affinity $U$ for $f$. Then $f(x) \geq \lambda(x)$ for all $x \in S$.

Proof. Pick $y$ in the topological interior of $U$ relative to the affine hull of $S$; then for $t \in[0,1]$ sufficiently close to $0, z=t x+(1-t) y$ will also belong to $U$. The claim now follows from (1.6.1).

Corollary 3.8. With notation as in Lemma 3.7, the maximal domain of affinity $U$ with ambient functional $\lambda$ is a convex set.

Proof. For $x, y \in U$ and $t \in[0,1]$, for $z=t x+(1-t) y$, we have

$$
f(z) \geq \lambda(z)=t \lambda(x)+(1-t) \lambda(y)=t f(x)+(1-t) f(y) \geq f(z) .
$$

Hence $z \in U$.

We build domains of affinity using the following argument.

Lemma 3.9. Let $T \subseteq \mathbb{R}^{n}$ be a subset with convex hull $U$, and pick $z \in \operatorname{int}(U)$. Let $f: U \rightarrow \mathbb{R}$ be a convex function, let $\lambda: \mathbb{R}^{n} \rightarrow \mathbb{R}$ be an affine functional, and suppose that $f(x)=\lambda(x)$ for all $x \in T \cup\{z\}$. Then $f(x)=\lambda(x)$ for all $x \in U$.

Proof. Pick any $x \in U$. On one hand, by (1.6.2), $f(x) \leq \lambda(x)$. On the other hand, since $z \in \operatorname{int}(U)$, there exist $y \in U$ and $t \in(0,1]$ such that $t x+(1-t) y=z$ (namely, pick $y$ on the ray from $z$ away from $x$ ). Since $y$ is in the convex hull of $T$, we can find $y_{1}, \ldots, y_{m} \in T$ and $t_{1}, \ldots, t_{m} \in[0,1]$ with $t_{1}+\cdots+t_{m}=1$, such that $t_{1} y_{1}+\cdots+t_{m} y_{m}=y$. Then

$$
t x+(1-t) t_{1} y_{1}+\cdots+(1-t) t_{m} y_{m}=z,
$$

so (1.6.2) implies $f(x) \geq \lambda(x)$. 


\section{Polyhedral Sets and Functions}

Definition 4.1. Let $F$ be a subfield of $\mathbb{R}$. An (F-rational $)$ closed half-space is a subset of $\mathbb{R}^{n}$ of the form

$$
S=\left\{x \in \mathbb{R}^{n}: \lambda(x) \geq 0\right\}
$$

for $\lambda$ an (F-integral) affine functional with nonzero slope. A subset $S \subseteq \mathbb{R}^{n}$ is ( $F$-rational) polyhedral if it is the intersection of some finite number of ( $F$-rational) closed half-spaces. (This number may be zero, in which case $S=\mathbb{R}^{n}$.) A polyhedral subset of $\mathbb{R}^{n}$ is closed and convex, but not necessarily bounded. We use rational and transrational as synonyms for $\mathbb{Q}$-rational and $\mathbb{R}$-rational, respectively.

Lemma 4.2. Let $S \subseteq \mathbb{R}^{n}$ be a polyhedral subset. Then any convex function $f$ : $S \rightarrow \mathbb{R}$ is upper semicontinuous. Consequently, $f$ is continuous if and only if it is lower semicontinuous.

Proof. See [7, Theorem 10.2].

Definition 4.3. Let $S \subseteq \mathbb{R}^{n}$ be a polyhedral subset. Choose affine functionals $\lambda_{1}, \ldots, \lambda_{m}: \mathbb{R}^{n} \rightarrow \mathbb{R}$ such that

$$
S=\left\{x \in \mathbb{R}^{n}: \lambda_{i}(x) \geq 0(i=1, \ldots, m)\right\} .
$$

A facet of $S$ is a nonempty subset of $S$ of the form

$$
B=\left\{x \in S: \lambda_{i}(x)=0(i \in I)\right\}
$$

for some subset $I$ of $\{1, \ldots, m\}$; this definition does not depend on the choice of $\lambda_{1}, \ldots, \lambda_{m}$. A facet is proper if it is not equal to $S$. Note that the union of the proper facets of $S$ equals $S \backslash \operatorname{int}(S)$. An element $x \in S$ forming a facet by itself is called a vertex of $S$.

Definition 4.4. For $S \subseteq \mathbb{R}^{n}$, a function $f: S \rightarrow \mathbb{R}$ is $(G$-integral) polyhedral if it has the form

$$
f(x)=\max \left\{\lambda_{1}(x), \ldots, \lambda_{m}(x)\right\} \quad(x \in S)
$$

for some (G-integral) affine functionals $\lambda_{1}, \ldots, \lambda_{m}: \mathbb{R}^{n} \rightarrow \mathbb{R}$. This implies that $f$ is convex and continuous.

Lemma 4.5. Let $S \subseteq \mathbb{R}^{n}$ be a polyhedral subset. Let $f: S \rightarrow \mathbb{R}$ be a function. Then the following conditions are equivalent.

(a) $f$ is polyhedral.

(b) $f$ is convex and $S$ is covered by finitely many domains of affinity for $f$.

(c) $f$ is convex and $S$ is covered by domains of affinity for $f$ whose ambient functionals have only finitely many different slopes. 
Proof. If $f(x)=\sup _{i}\left\{\lambda_{i}(x)\right\}$ with each $\lambda_{i}$ affine, then it is clear that $f$ is convex and that the domains of affinity for $f$ corresponding to the $\lambda_{i}(x)$ cover $S$. Hence (a) implies (b). Conversely, if $f$ is convex and $S$ is covered by domains of affinity for $f$ with ambient functionals $\lambda_{1}, \ldots, \lambda_{m}$, then $f(x)=\sup _{i}\left\{\lambda_{i}(x)\right\}$ by Lemma 3.7. Hence (b) implies (a).

It is trivial that (b) implies (c). The reverse implication holds by Lemma 3.7, which implies that no domains of affinity for $f$ can have distinct ambient functionals with the same slope.

Definition 4.6. Let $S \subseteq \mathbb{R}^{n}$ be a convex subset. A function $f: S \rightarrow \mathbb{R}$ is locally (G-integral) polyhedral if for each $x \in S$, there exists a neighborhood $U$ of $x$ in $\mathbb{R}^{n}$ such that the restriction of $f$ to $U \cap S$ is ( $G$-integral) polyhedral. If $f$ is locally polyhedral, then $f$ is evidently continuous; by replacing $\mathbb{R}^{n}$ with the affine hull of $S$ and applying Lemma 3.3 on $\operatorname{int}(S)$, we see that $f$ is also convex.

Lemma 4.7. Let $S \subseteq \mathbb{R}^{n}$ be a compact convex subset. Then $f: S \rightarrow \mathbb{R}$ is locally polyhedral if and only if $f$ is polyhedral.

Proof. Suppose $f$ is locally polyhedral. Then each $x \in S$ admits a neighborhood $U$ in $\mathbb{R}^{n}$ such that the restriction of $f$ to $U \cap S$ is polyhedral. Since $S$ is compact, it is covered by finitely many of these neighborhoods. Hence $S$ is covered by finitely many domains of affinity for $f$; by Lemma $4.5, f$ is polyhedral.

\section{Extending Convex Functions}

It is useful to note that the notions of $F$-convexity and ordinary convexity are essentially equivalent; this refines the usual assertion that a convex function on an open convex set is continuous [7, Theorem 10.1].

Theorem 5.1. Let $F$ be a subfield of $\mathbb{R}$. Let $S \subseteq \mathbb{R}^{n}$ be an open convex subset. Let $f: S \cap F^{n} \rightarrow \mathbb{R}$ be a function such that for each $x \in F^{n}$ and each $i \in\{1, \ldots, n\}$, the restriction of $f$ to $S \cap F^{n} \cap\left(x+\mathbb{R} e_{i}\right)$ is $F$-convex.

(a) The function $f$ extends uniquely to a continuous function $\tilde{f}$ on $S$ such that for each $x \in \mathbb{R}^{n}$ and each $i \in\{1, \ldots, n\}$, the restriction of $\tilde{f}$ to $S \cap\left(x+\mathbb{R} e_{i}\right)$ is convex.

(b) If $f$ is $F$-convex, then $\tilde{f}$ is convex.

(c) For any closed intervals $I_{1}, \ldots, I_{n}$ such that $I_{1} \times \cdots \times I_{n} \subset S$, the directional derivatives $f^{\prime}\left(x, \pm e_{i}\right)$ for $x \in I_{1} \times \cdots \times I_{n}$ and $i \in\{1, \ldots, n\}$ are bounded.

Proof. We proceed by induction on $n$, with trivial base case $n=0$. Pick $x=$ $\left(x_{1}, \ldots, x_{n}\right) \in S$ (not necessarily in $\left.S \cap F^{n}\right)$. For $i=1, \ldots, n$, pick closed intervals $I_{i, 0}, I_{i, 1}$ with endpoints in $F$, such that $x_{i}$ is contained in the interior of $I_{i, 0}, I_{i, 0}$ is contained in the interior of $I_{i, 1}$, and $I_{1,1} \times \cdots \times I_{n, 1} \subset S$. 
Put $B_{j}=I_{1, j} \times \cdots \times I_{n, j}$ for $j=0,1$. By the induction hypothesis, $f$ extends uniquely to a continuous function on each face of each box $B_{j}$. In particular, $f$ achieves maximum and minimum values on the boundary $\partial\left(B_{j}\right)$ of each $B_{j}$.

We now argue that $f$ is Lipschitz continuous on $B_{0}$. Pick any $y, z \in B_{0} \cap F^{n}$ whose difference is a nonzero multiple of some $e_{i}$. Then there exist a unique affine function $g: \mathbb{R} \rightarrow \mathbb{R}^{n}$ and values $a_{1}<0 \leq a_{0}<b_{0} \leq 1<b_{1}$, all contained in $F$, such that

$$
\begin{aligned}
g\left(a_{0}\right) & =y, \\
g\left(b_{0}\right) & =z, \\
\left\{t \in \mathbb{R}: g(t) \in B_{0}\right\} & =[0,1], \\
\left\{t \in \mathbb{R}: g(t) \in B_{1}\right\} & =\left[a_{1}, b_{1}\right] .
\end{aligned}
$$

We have $g\left(a_{1}\right), g(0), g(y), g(z), g(1), g\left(b_{1}\right) \in F^{n}$, so the $F$-convexity of the restriction of $f$ to the image of $g$ implies

$$
\frac{g(0)-g\left(a_{1}\right)}{-a_{1}} \leq \frac{g(z)-g(y)}{b_{0}-a_{0}} \leq \frac{g\left(b_{1}\right)-g(1)}{b_{1}-1} .
$$

In this expression, the quantities $g\left(a_{1}\right), g(0), g(1), g\left(b_{1}\right)$ are bounded because $f$ is bounded on $\partial\left(B_{0}\right) \cup \partial\left(B_{1}\right)$. The quantities $-a_{1}$ and $b_{1}-1$ depend only on $i$, so they are bounded above and bounded below away from 0 .

It now follows that for some $c>0$, for all $y=\left(y_{1}, \ldots, y_{n}\right), z=\left(z_{1}, \ldots, z_{n}\right) \in$ $B_{0} \cap F^{n}$, we have

$$
|f(y)-f(z)| \leq c\left(\left|y_{1}-z_{1}\right|+\cdots+\left|y_{n}-z_{n}\right|\right) .
$$

This implies that $f$ is Lipschitz continuous on $B_{0}$, so $f$ extends uniquely to a continuous function on $B_{0}$.

Since $B_{0}$ was chosen to contain an arbitrary $x \in S$ in its interior, we conclude that $f$ extends uniquely to a continuous function on all of $S$, proving (a). We deduce (b) as an immediate corollary because the terms of (1.6.1) vary continuously with the arguments. We deduce (c) from the Lipschitz property established above.

\section{Detecting Locally Polyhedral Functions}

We now give a criterion for local $F$-integral polyhedrality.

Theorem 6.1. Let $F$ be a subfield of $\mathbb{R}$. Let $S \subseteq \mathbb{R}^{n}$ be an open convex subset. Let $f: S \cap F^{n} \rightarrow \mathbb{R}$ be a continuous function. Suppose that the restriction of $f$ to every F-rational line segment contained in $S$ and parallel to one of the coordinate axes is F-integral polyhedral. Then the continuous extension of $f$ to $S$ given by Theorem 5.1 is locally F-integral polyhedral.

The proof will be by induction on $n$, with trivial base case $n=1$. In order to break the proof up into a sequence of lemmas, we must assert a hypothesis that will be available during the induction step. 
Hypothesis 6.2. Fix a value of $n$, and set notation as in Theorem 6.1. By Theorem 5.1, $f$ extends uniquely to a continuous function whose restriction to each line parallel to a coordinate axis is convex; we will also call this extended function $f$. Also assume that the conclusion of Theorem 6.1 holds in all cases where $n$ is replaced by any smaller positive integer.

Lemma 6.3. Let $I \subseteq \mathbb{R}$ be an open subinterval. Let $f: I \rightarrow \mathbb{R}$ be a convex function such that $f^{\prime}(x, 1) \in \mathbb{Z}$ for all $x \in \mathbb{Q}$. Then $f$ is locally transintegral polyhedral.

Proof. For any two $x_{1}, x_{2} \in I$ for which $f^{\prime}\left(x_{1}, 1\right)=f^{\prime}\left(x_{2}, 1\right)$ and any $x_{3} \in\left(x_{1}, x_{2}\right)$, we have

$$
\begin{aligned}
f^{\prime}\left(x_{1}, 1\right) & \leq \frac{f\left(x_{3}\right)-f\left(x_{1}\right)}{x_{3}-x_{1}} \\
& \leq \frac{f\left(x_{2}\right)-f\left(x_{3}\right)}{x_{2}-x_{3}} \\
& \leq f^{\prime}\left(x_{2}, 1\right)=f^{\prime}\left(x_{1}, 1\right) .
\end{aligned}
$$

Hence $f$ is affine with integral slope on $\left[x_{1}, x_{2}\right]$.

Let $J=[a, b]$ be any closed subinterval of $\operatorname{int}(I)$. The values $f^{\prime}(x, 1)$ for $x \in I$ are integral and lie in the finite interval $\left[f^{\prime}(a, 1), f^{\prime}(b, 1)\right]$, so they are restricted to a finite set. Since $f^{\prime}(x, 1)$ is nondecreasing, the set of $x$ for which $f^{\prime}(x, 1)$ takes any particular value is connected. On such a set, by the previous paragraph $f$ is affine with integral slope. The closures of these sets cover $J$, so $f$ is transintegral polyhedral on $J$. This proves that $f$ is locally transintegral polyhedral on $I$.

Lemma 6.4. Under Hypothesis 6.2 , for any $x \in S$ and any $i \in\{1, \ldots, n\}$, the restriction of $f$ to $x+\mathbb{R} e_{i}$ is locally transintegral polyhedral.

Proof. It suffices to treat the case $i=n$. Write $x=\left(x_{1}, \ldots, x_{n}\right)$. By Lemma 6.3, it suffices to check that if $x_{n} \in F$, then $f^{\prime}\left(x, e_{n}\right) \in \mathbb{Z}$.

Let $H$ denote the hyperplane spanned by $e_{1}, \ldots, e_{n-1}$. Since $x_{n} \in F$, the hyperplane $x+H$ is $F$-rational. By Hypothesis 6.2, the restriction of $f$ to $x+H$ is locally $F$-integral polyhedral. Consequently, there exists an $F$-rational polyhedral subset $U$ of $x+H$ such that $x \in \operatorname{int}(U)$ and $f$ is affine on $U$. The set $\operatorname{int}(U) \cap F^{n}$ is dense in $U$ because $U$ is $F$-rational polyhedral. By Lemma 2.2, the function $f^{\prime}\left(y, e_{n}\right)$ is $F$-convex for $y \in \operatorname{int}(U) \cap F^{n}$; on the other hand, for $y \in \operatorname{int}(U) \cap F^{n}$, we have $f^{\prime}\left(y, e_{n}\right) \in \mathbb{Z}$ by Hypothesis 6.2 . Hence $f^{\prime}\left(y, e_{n}\right)$ is equal to a constant integer value on all of $\operatorname{int}(U)$. In particular, $f^{\prime}\left(x, e_{n}\right) \in \mathbb{Z}$, completing the proof.

Lemma 6.5. Under Hypothesis 6.2, $f$ is convex and each $x \in S$ belongs to a domain of affinity for $f$ whose ambient functional is transintegral.

Proof. By Lemma 6.4, we may reduce to the case $F=\mathbb{R}$. We will use the criterion of Lemma 3.3 to prove convexity; it suffices to show that for each $x \in S$, we can find 
a neighborhood $U$ of $x$ in $S$ and a convex function $g: U \rightarrow \mathbb{R}$ such that $f(x)=g(x)$ and $f(y) \geq g(y)$ for $y \in U$. In the process, we will exhibit a domain of affinity for $f$ containing $x$.

Let $H$ denote the hyperplane spanned by $e_{1}, \ldots, e_{n-1}$. By Hypothesis 6.2 , the restriction of $f$ to $x+H$ is locally transintegral polyhedral. In particular, of the domains of affinity for $f$ on $S \cap(x+H)$, only finitely many contain $x$. Call these $V_{1}, \ldots, V_{m}$, and let $\lambda_{1}, \ldots, \lambda_{m}: x+H \rightarrow \mathbb{R}$ be the corresponding ambient functionals; then by Lemma 3.7,

$$
f(y) \geq \sup \left\{\lambda_{1}(y), \ldots, \lambda_{m}(y)\right\} \quad(y \in S \cap(x+H))
$$

with equality at $y=x$.

For $j=1, \ldots, m$, extend $\lambda_{j}$ to an affine functional on $\mathbb{R}^{n}$ which is constant on $\mathbb{R} e_{n}$. Pick any $z_{j} \in \operatorname{int}\left(V_{j}\right)$, and define a function $\mu_{j}:(S \cap(x+H))+\mathbb{R} e_{n} \rightarrow \mathbb{R}$ by setting

$$
\mu_{j}\left(y+t e_{n}\right)=\left\{\begin{array}{ll}
\lambda_{j}(y)+t f^{\prime}\left(z_{j}, e_{n}\right) & (t \geq 0) \\
\lambda_{j}(y)-t f^{\prime}\left(z_{j},-e_{n}\right) & (t \leq 0)
\end{array} \quad(y \in S \cap(x+H)) .\right.
$$

This definition does not depend on the choice of $z_{j}$, by an argument as in the proof of Lemma 6.4: $f^{\prime}\left(z_{j}, \pm e_{n}\right)$ takes integer values but is convex in $z_{j}$ by Lemma 2.2 , so must be constant on $\operatorname{int}\left(V_{j}\right)$. Moreover, for $\epsilon>0$ sufficiently small, we may choose $z_{j, 1}, \ldots, z_{j, n+1} \in \operatorname{int}\left(V_{j}\right)$ with $z_{j, n+1}$ in the interior of the simplex with vertices $z_{j, 1}, \ldots, z_{j, n}$, such that for $k=1, \ldots, n+1, f\left(z_{j, k}+t e_{n}\right)$ is affine for $t \in[0, \epsilon]$. Then the convexity of $f$ on $S \cap\left(x+H+t e_{n}\right)$ (given by Hypothesis 6.2) and Lemma 3.9 imply that for $t \in[0, \epsilon]$, the restriction of $f$ to $x+H+t e_{n}$ admits a domain of affinity with ambient functional $\lambda_{j}(y)+t f^{\prime}\left(z_{j}, e_{n}\right)$. By Lemma 3.7, $f(y) \geq \mu_{j}(y)$ for $y \in S \cap\left(x+H+t e_{n}\right)$. By arguing similarly for negative $t$, we conclude that for some $\epsilon>0$,

$$
f(y) \geq \sup \left\{\mu_{1}(y), \ldots, \mu_{m}(y)\right\} \quad\left(y \in S \cap\left((x+H)+[-\epsilon, \epsilon] e_{n}\right)\right),
$$

with equality at $y=x$.

By Hypothesis 6.2 , the restriction of $f$ to $S \cap\left(z_{j}+\mathbb{R} e_{n}\right)$ is convex, so $f^{\prime}\left(z_{j}, e_{n}\right) \geq$ $-f^{\prime}\left(z_{j},-e_{n}\right)$. Hence $\mu_{j}$ is convex, so (6.5.1) implies that the criterion of Lemma 3.3 is satisfied at $x$. Since $x \in S$ was arbitrary, we deduce that $f$ is convex. Moreover, for each $j$,

$$
\left(y_{1}, \ldots, y_{n}\right) \mapsto \lambda_{j}\left(y_{1}, \ldots, y_{n-1}\right)+f^{\prime}\left(z_{j}, e_{n}\right) y_{n}
$$

is a transintegral affine functional which agrees with $f$ both at $x$ and on an open subset of $S$, so its corresponding domain of affinity contains $x$.

We are now ready to prove Theorem 6.1.

Proof of Theorem 6.1. We induct on $n$ with trivial base case $n=1$. Under the induction hypothesis, $f$ extends continuously to $S$ by Theorem 5.1 , and this extended function is convex by Lemma 6.5 . 
We first check that $f$ is polyhedral on any closed box $B$ contained in $S$. By Lemma $6.5, B$ is covered by domains of affinity for $f$ whose ambient functionals are transintegral; by Theorem 5.1, the slopes of these functionals are bounded. Hence there can be only finitely many slopes; by Lemma $4.5, f$ is polyhedral.

It now follows that $f$ is locally polyhedral. To check that each ambient functional is $F$-integral polyhedral, we simply restrict to some $F$-rational line segments of positive length parallel to $e_{1}, \ldots, e_{n}$ contained in the corresponding domain of affinity.

\section{Detecting Polyhedral Functions on Polyhedra}

One can ask also for criteria for detecting $F$-integral polyhedrality of a function. To articulate results as strong as possible in this direction, we assume that the function is already known to be locally $F$-integral polyhedral; then by Lemma 4.5, it suffices to check that there are only finitely many ambient functionals.

Lemma 7.1. Let $S \subseteq \mathbb{R}^{n}$ be a convex subset. Let $U \subseteq S$ be a convex subset. Suppose $z \in \mathbb{R}^{n}$ is such that for any $x \in U$, there exists $t>0$ for which $x+t z \in S$. Let $f: S \rightarrow \mathbb{R}$ be a locally polyhedral function whose restriction to $U$ is affine. Then $f^{\prime}(x, z)$ is constant for $x \in \operatorname{int}(U)$.

Proof. The function $f^{\prime}(x, z)$ is convex on $\operatorname{int}(U)$ by Lemma 2.2. On the other hand, since $f$ is locally polyhedral, $f^{\prime}(x, z)$ is locally limited to a finite set. Hence $f^{\prime}(x, z)$ must be constant on $\operatorname{int}(U)$. (Compare the proof of Lemma 6.4.)

Definition 7.2. An affine orthant in $\mathbb{R}^{n}$ is a subset of $\mathbb{R}^{n}$ of the form

$$
\left\{x \in \mathbb{R}^{n}: \lambda_{i}(x) \geq 0 \quad(i=1, \ldots, n)\right\},
$$

for some affine functionals $\lambda_{1}, \ldots, \lambda_{n}$ whose slopes are linearly independent.

Lemma 7.3. Let $S \subseteq \mathbb{R}^{n}$ be a polyhedral subset with affine hull $\mathbb{R}^{n}$, for some $n \geq 2$. Assume that $S$ is contained in an affine orthant. Let $f: S \rightarrow \mathbb{R}^{n}$ be a continuous convex function. Suppose that the restriction of $f$ to $\operatorname{int}(S)$ is locally transintegral polyhedral, and that the restriction of $f$ to each proper facet of $S$ is polyhedral. Then $f$ is transintegral polyhedral.

Proof. Since the restriction of $f$ to each proper facet of $S$ is polyhedral, we can partition $S \backslash \operatorname{int}(S)$ into finitely many subsets $P_{1}, \ldots, P_{k}$, such that each $P_{i}$ is the interior of a polyhedral subset contained in some proper facet, and $f$ is affine on each $P_{i}$.

Suppose $S$ is contained in the affine orthant defined by the affine functionals $\lambda_{1}, \ldots, \lambda_{n}$ with slopes $\mu_{1}, \ldots, \mu_{n}$. Let $z_{1}, \ldots, z_{n}$ be a basis of $\mathbb{R}^{n}$ such that for each $i \in\{1, \ldots, n\}$, the quantities $\mu_{1}\left(z_{i}\right), \ldots, \mu_{n}\left(z_{i}\right)$ are all nonzero and not all of the same sign. 
Suppose $i \in\{1, \ldots, n\}, j \in\{1, \ldots, k\}, x_{1}, x_{2} \in P_{j}$ are such that for some $i$, $f^{\prime}\left(x_{1}, z_{i}\right)$ and $f^{\prime}\left(x_{2}, z_{i}\right)$ are both defined. Then there is an open convex set $U \subseteq P_{j}$ containing both $x_{1}$ and $x_{2}$, such that $f^{\prime}\left(x, z_{i}\right)$ is defined for all $x \in U$. By Lemma 7.1, $f^{\prime}\left(x_{1}, z_{i}\right)=f^{\prime}\left(x_{2}, z_{i}\right)$.

Now pick any $x \in S$ and put $I_{i}=\left\{t \in \mathbb{R}: x+t z_{i} \in S\right\}$. By the choice of $z_{i}$, the interval $I_{i}$ is always bounded, so we can write it as $[a, b]$. By the previous paragraph, $f^{\prime}\left(x+a z_{i}, z_{i}\right)$ and $f^{\prime}\left(x+b z_{i},-z_{i}\right)$ are both limited to finite sets. By convexity, this limits $f^{\prime}\left(x, z_{i}\right)$ to a bounded interval.

Applying this for $i=1, \ldots, n$, we deduce that for any ambient functional $\lambda$ for $f$ with slope $\mu, \mu\left(z_{1}\right), \ldots, \mu\left(z_{n}\right)$ are limited to bounded intervals. Since $\mu$ is integral, this limits $\mu$ to a finite set. By Lemma $4.5, f$ is polyhedral, as desired.

At this point, we can already recover a result of the first author and Liang Xiao [6, Theorem 3.2.4], by specializing the following theorem to the case $F=\mathbb{R}$.

Theorem 7.4. Let $F$ be a subfield of $\mathbb{R}$. Let $S$ be an $F$-rational polyhedral subset. Let $f: S \cap F^{n} \rightarrow \mathbb{R}$ be a function whose restriction to any $F$-rational line is $F$-integral polyhedral. Then $f$ is F-integral polyhedral.

Proof. By Theorem 5.1, the restriction of $f$ to $\operatorname{int}(S)$ extends uniquely to a continuous convex function $g: \operatorname{int}(S) \rightarrow \mathbb{R}$. By Theorem $6.1, g$ is locally $F$-integral polyhedral on $\operatorname{int}(S)$. Use the formula (3.4.1) to extend $g$ to a lower semicontinuous convex function $g: S \rightarrow \mathbb{R}$; then $g$ is continuous on $S$ by Lemma 4.2. For each $F$-rational line $L$ meeting $\operatorname{int}(S), f$ and $g$ agree on $\operatorname{int}(S) \cap F^{n} \cap L$. In particular, both functions are $F$-integral polyhedral on $\operatorname{int}(S) \cap F^{n} \cap L=\operatorname{int}(S \cap L) \cap F^{n}$. Since $f$ is also $F$-integral polyhedral on $S \cap F^{n} \cap L$ while $g$ is continuous on that same domain, we conclude that $f$ and $g$ agree on $S \cap F^{n} \cap L$. Since each element of $S \cap F^{n}$ lies on an $F$-rational line meeting $\operatorname{int}(S)$, we conclude that $f$ and $g$ agree on all of $S \cap F^{n}$.

To prove that $f$ is $F$-integral polyhedral, it now suffices to prove that $g$ is $F$-integral polyhedral. It suffices to check this with $S$ replaced by $S \cap$ $\left([0,+\infty) c_{1} e_{1}+\cdots+[0,+\infty) c_{n} e_{n}\right)$ for each $\left(c_{1}, \ldots, c_{n}\right) \in\{ \pm 1\}^{n}$. In each of these cases, we may deduce the claim by induction on $\operatorname{dim}(S)$, using Theorem 7.3.

We next prove a much stronger form of Theorem 7.4.

Lemma 7.5. Assume one of the following sets of hypotheses.

(a) Let $x_{1}, x_{2}, x_{3} \in \mathbb{R}^{2}$ be distinct points such that the segment $x_{2} x_{3}$ is transrational. Let $\ell_{1}, \ell_{2}$ denote the segments $x_{1} x_{2}, x_{2} x_{3}$.

(b) Let $x_{1}, x_{2} \in \mathbb{R}^{2}$ be distinct points. Let $\ell_{1}, \ell_{2}$ be parallel closed transrational rays emanating from $x_{1}, x_{2}$.

Let $S$ be the convex hull of $x_{1} x_{2} \cup \ell_{1} \cup \ell_{2}$. Suppose that $f: S \rightarrow \mathbb{R} \cup\{+\infty\}$ is a lower semicontinuous convex function such that the restriction of $f$ to $\operatorname{int}(S)$ (takes 
finite values and) is locally transintegral polyhedral, and the restrictions of $f$ to $x_{1} x_{2}$ and $\ell_{1}$ (take finite values and) are polyhedral. Then $f$ takes finite values and is polyhedral.

Proof. Let $z \in \mathbb{Q}^{2}$ be a nonzero vector parallel to $\ell_{2}$ in the direction emanating from $x_{2}$. We check that for $x \in S \backslash \ell_{2}, f^{\prime}(x, z)$ is limited to a bounded set independent of $x$.

(a) In this case, we may argue just as in Lemma 7.3: there exist $a \leq b \in \mathbb{R}$ with $x+a z \in x_{1} x_{2}$ and $x+b z \in \ell_{1}, f^{\prime}(x, z)$ is trapped between $f^{\prime}(x+a z, z)$ and $-f^{\prime}(x+b z,-z)$ by convexity, and each of those is limited to a finite set.

(b) As in (a), we see that $f^{\prime}(x, z)$ is bounded below. Put

$$
P=\left\{(a, b) \in \mathbb{R}^{2}: 0 \leq a, 0 \leq b, a+b \leq 1\right\} .
$$

Define the bijection $h: P \backslash\{(1,0)\} \rightarrow S$ by

$$
h(a, b)=x_{1}+\frac{a}{2-2 a-b} z+\frac{b}{2-2 a-b}\left(x_{2}-x_{1}\right) .
$$

Then for any affine functional $\lambda$ on $\mathbb{R}^{2},(2-2 a-b) \lambda \circ h$ is again an affine functional on $\mathbb{R}^{2}$ (although transintegrality may not be preserved). Consequently, $F=(2-2 a-b) f \circ h$ is locally polyhedral on $\operatorname{int}(P)$ and polyhedral on the horizontal and vertical proper facets of $P$. Using the formula (3.4.1), we may extend $F$ to a lower semicontinuous convex function $F: P \rightarrow \mathbb{R} \cup\{+\infty\}$. Since $F$ is bounded on a subset (the union of the horizontal and vertical facets) with convex hull $P, F$ takes finite values everywhere. Hence $F$ is continuous by Lemma 4.2 .

For any $c, d \geq 0$ not both zero with $d<c$, we have

$$
\begin{aligned}
F(1,0) & =\lim _{s \rightarrow 0^{+}} F(1-s c, s d) \\
& =\lim _{s \rightarrow 0^{+}}(2 s c-s d) f\left(x_{1}+\frac{1-s c}{2 s c-s d} z+\frac{s d}{2 s c-s d}\left(x_{2}-x_{1}\right)\right) .
\end{aligned}
$$

If $\lambda$ is an ambient functional for $f$ with slope $\mu$, by Lemma 3.7 we have

$$
\begin{aligned}
F(1,0) & \geq \lim _{s \rightarrow 0^{+}}(2 s c-s d) \lambda\left(x_{1}+\frac{1-s c}{2 s c-s d} z+\frac{s d}{2 s c-s d}\left(x_{2}-x_{1}\right)\right) \\
& =\lim _{s \rightarrow 0^{+}} \mu\left((1-s c) z+s d\left(x_{2}-x_{1}\right)\right) \\
& =\mu(z) .
\end{aligned}
$$

Hence $\mu(z)$ is bounded above, as then is $f^{\prime}(x, z)$ for any $x \in S \backslash \ell_{2}$.

Since $f$ is locally transintegral polyhedral and $z \in \mathbb{Q}^{2}, f^{\prime}(x, z)$ is in fact limited to a finite set $T$. For $m \in T$ and $t \in[0,1)$, define

$$
g_{m}(t)=\inf _{u}\left\{f\left(x_{1}+t\left(x_{2}-x_{1}\right)+u z\right)-m u\right\} .
$$


A straightforward calculation shows that $g_{m}(t)$ is convex, as follows. Given $t_{1}, t_{2} \in$ $[0,1)$ and $w \in[0,1]$, put $t_{3}=w t_{1}+(1-w) t_{2}$. For any $\delta>0$, we may find $u_{1}, u_{2}$ with

$$
f\left(x_{1}+t_{i}\left(x_{2}-x_{1}\right)+u_{i} z\right)-m u_{i} \leq g_{m}\left(t_{i}\right)+\delta \quad(i=1,2) ;
$$

then for $u_{3}=w u_{1}+(1-w) u_{2}$, we have

$$
\begin{aligned}
w g_{m}\left(t_{1}\right)+(1-w) g_{m}\left(t_{2}\right) \geq & w\left(f\left(x_{1}+t_{1}\left(x_{2}-x_{1}\right)+u_{1} z\right)-m u_{1}\right) \\
& +(1-w)\left(f\left(x_{1}+t_{2}\left(x_{2}-x_{1}\right)+u_{2} z\right)-m u_{2}\right)-\delta \\
\geq & f\left(x_{1}+t_{3}\left(x_{2}-x_{1}\right)+u_{3} z\right)-m u_{3}-\delta \\
\geq & g_{m}\left(t_{3}\right)-\delta .
\end{aligned}
$$

Since $\delta>0$ was arbitrary, $g_{m}(t)$ must be convex.

We may extend $g_{m}(t)$ to a lower semicontinuous convex function $g_{m}:[0,1] \rightarrow$ $\mathbb{R} \cup\{+\infty\}$ using (3.4.1). Note that $g_{m}(t) \leq f\left(x_{1}+t\left(x_{2}-x_{1}\right)\right) \leq \max \left\{f\left(x_{1}\right), f\left(x_{2}\right)\right\}$ for all $t \in[0,1)$, so $g_{m}(1)<+\infty$. By Lemma $4.2, g_{m}:[0,1] \rightarrow \mathbb{R}$ is continuous.

For $t \in[0,1)$ and $u$ such that $x_{1}+t\left(x_{2}-x_{1}\right)+u z \in S$, we have

$$
f\left(x_{1}+t\left(x_{2}-x_{1}\right)+u z\right)=\sup _{m \in T}\left\{g_{m}(t)+m u\right\} .
$$

The right side of (7.5.1) extends to a continuous convex function of $x_{1}+t\left(x_{2}-\right.$ $\left.x_{1}\right)+u z$ on all of $S$ with finite values. Since the left side of (7.5.1) is convex and lower semicontinuous, it must also take finite values; hence both sides of (7.5.1) are continuous by Lemma 4.2, and thus must coincide.

The right side of (7.5.1), when restricted to $t=1$, is polyhedral. Hence $f$ is polyhedral on $\ell_{2}$, so Lemma 7.3 implies that $f$ is polyhedral on $S$.

Theorem 7.6. Let $F$ be a subfield of $\mathbb{R}$. Let $S \subseteq \mathbb{R}^{n}$ be an $F$-rational polyhedral subset contained in an affine orthant. Let $f: \operatorname{int}(S) \rightarrow \mathbb{R}$ be a locally F-integral polyhedral function. Suppose that there exist $\ell_{1}, \ldots, \ell_{m}$ with the following properties.

(a) For $i=1, \ldots, m, \ell_{i}$ is a convex subset of a line (not necessarily F-rational) and $\operatorname{int}\left(\ell_{i}\right) \subseteq \operatorname{int}(S)$.

(b) For $i=1, \ldots, m, f$ is polyhedral on $\operatorname{int}\left(\ell_{i}\right)$.

(c) For each vertex $Q$ of $S$, there is some $i$ such that $Q \in \ell_{i}$.

(d) For each unbounded one-dimensional facet $B$ of $S$, there is some $i$ such that $\ell_{i}$ is a translate of $B$.

Then $f$ is polyhedral.

Proof. We immediately reduce to the case where $\operatorname{dim}(S)=n$. If $n=1$, then the $\ell_{i}$ must cover $S$ and so $f$ is automatically polyhedral. We thus assume $n \geq 2$ hereafter.

Extend $f$ to a lower semicontinuous function $f: S \rightarrow \mathbb{R} \cup\{+\infty\}$ using (3.4.1). Let $y$ be any vertex of $S$. By hypothesis, one of the $\ell_{i}$ has endpoint $y$. Pick $x \in$ $\operatorname{int}\left(\ell_{i}\right)$; then $x \in \operatorname{int}(S)$. Then for any $z \in \operatorname{int}(S)$ such that $y z$ is $F$-rational, we may apply Lemma 7.5 to deduce that $f$ is polyhedral on $y z$. 
Let $B$ be a one-dimensional facet of $S$. Since $S$ is contained in an affine orthant, $B$ has at least one endpoint $y$.

(a) If $B$ is bounded, let $z$ be its other endpoint. Choose a two-dimensional $F$ rational plane containing $B$ and passing through $\operatorname{int}(S)$. We can find a point $x$ on this plane so that $x \in \operatorname{int}(S)$, and $x y$ and $x z$ are both $F$-rational. We have from above that $f$ is polyhedral on $x y$ and $x z$; it is thus polyhedral on $B$ by Lemma 7.5.

(b) If $B$ is unbounded, pick any $x \in \operatorname{int}(S)$ such that $x y$ is $F$-rational. By hypothesis, one of the $\ell_{i}$ is a translate of $B$. Let $R$ be the ray from $x$ parallel to $B$; then by Lemma 7.5 (applied to $x$ and $\ell_{i}$ ), $f$ is polyhedral on $R$. We have from above that $f$ is polyhedral on $x y$; thus by Lemma 7.5 again, $f$ is polyhedral on $B$.

In either case, $f$ is polyhedral on each one-dimensional facet of $S$. By Lemma 7.3 and induction on dimension, $f$ is polyhedral on all of $S$, as desired.

As an immediate corollary, we now obtain a much stronger form of Theorem 7.4.

Corollary 7.7. Let $F$ be a subfield of $\mathbb{R}$. Let $S$ be an $F$-rational polyhedral subset. Let $f: \operatorname{int}(S) \cap F^{n} \rightarrow \mathbb{R}$ be a function whose restriction to any $F$-rational line is $F$-integral polyhedral. Then $f$ is F-integral polyhedral.

The difference between this result and Theorem 7.4 is that we do not assume anything about lines contained in the boundary of $S$.

Proof. Extend $f$ to a continuous convex function on $\operatorname{int}(S)$ using Theorem 5.1. By Theorem $6.1, f$ is locally $F$-integral polyhedral; by Theorem $7.6, f$ is polyhedral.

It should also be possible to formulate Theorem 7.6 without assuming that $S$ lies in an affine orthant. We leave the following as an exercise.

Exercise 7.8. Let $F$ be a subfield of $\mathbb{R}$. Let $S \subseteq \mathbb{R}^{n}$ be an $F$-rational polyhedral subset. Let $f: \operatorname{int}(S) \rightarrow \mathbb{R}$ be a locally $F$-integral polyhedral function. Suppose that there exist $\ell_{1}, \ldots, \ell_{m}$ with the following properties.

(a) For $i=1, \ldots, m, \ell_{i}$ is a convex subset of a line (not necessarily $F$-rational) and $\operatorname{int}\left(\ell_{i}\right) \subseteq \operatorname{int}(S)$.

(b) For $i=1, \ldots, m, f$ is polyhedral on $\operatorname{int}\left(\ell_{i}\right)$.

(c) The convex hull of $\ell_{1} \cup \cdots \cup \ell_{m}$ is equal to $S$.

Then $f$ is polyhedral.

\section{Integral Polyhedrality from Values}

If we restrict attention to integral polyhedral functions, we can do better than characterizing them by their restrictions to one-dimensional rational polyhedra. 
We can in fact identify them in terms of their restrictions to zero-dimensional rational polyhedra, i.e. to rational points, as long as we appropriately supplement with continuity and convexity hypotheses.

Lemma 8.1. Let $a \leq b$ be rational numbers. Let $f:[a, b] \rightarrow \mathbb{R}$ be a continuous convex function such that

$$
f(x) \in \mathbb{Z}+\mathbb{Z} x \quad(x \in(a, b) \cap \mathbb{Q}) .
$$

Then $f$ is integral polyhedral.

Proof. (Compare [3, Lemmas 2.3.1 and 2.4.1]) We first check that the restriction of $f$ to $(a, b)$ is locally integral polyhedral. Given $x \in(a, b) \cap \mathbb{Q}$, write $x=r / s$ in lowest terms. For $N$ any sufficiently large positive integer, we have

$$
\frac{f(x+1 /(s N))-f(x)}{1 /(s N)} \in s N\left(\mathbb{Z}+\mathbb{Z} x+\mathbb{Z}(s N)^{-1}\right)=\mathbb{Z} .
$$

As $N \rightarrow \infty$, this difference quotient runs through a sequence of integers which is nonincreasing (because $f$ is convex) and bounded below by $-f^{\prime}(x,-1)$ (because $x \in(a, b))$. Thus the quotient stabilizes for $N$ large. By convexity, the function $f$ must be affine with integral slope in a one-sided neighborhood of $x$. By Lemma 6.3, $f$ is locally transintegral polyhedral; by comparing values (e.g. for one irrational $x$ in each domain of affinity), we see that the constant terms must be in $\mathbb{Z}$. Hence $f$ is locally integral polyhedral.

We next check that $f$ is integral polyhedral. Suppose that the graph of $f$ has slopes which are unbounded below. The supporting lines of these slopes intersect the vertical line $x=a$ in points whose $y$-coordinates form a strictly increasing sequence within the discrete group $\mathbb{Z}+\mathbb{Z} a$. This sequence is bounded above by $f(a)$, contradiction.

That contradiction shows that the slopes of $f$ are bounded below; similarly, the slopes of $f$ are bounded above. Hence $f$ has only finitely many slopes on $(a, b)$, hence is integral polyhedral.

We now obtain the following result, which is a slight strengthening of [3, Theorem 2.4.2].

Theorem 8.2. Let $S \subset \mathbb{R}^{n}$ be a bounded rational polyhedral subset. Let $f: S \cap$ $\mathbb{Q}^{n} \rightarrow \mathbb{R}$ be a $\mathbb{Q}$-convex function whose restriction to each rational line segment is continuous, such that

$$
f\left(x_{1}, \ldots, x_{n}\right) \in \mathbb{Z}+\mathbb{Z} x_{1}+\cdots+\mathbb{Z} x_{n} \quad\left(\left(x_{1}, \ldots, x_{n}\right) \in S \cap \mathbb{Q}^{n}\right) .
$$

Then $f$ is integral polyhedral.

Proof. By Lemma 8.1, for each rational line $L$, the restriction of $f$ to $S \cap \mathbb{Q}^{n} \cap L$ is integral polyhedral. By Theorem 7.4, $f$ is $\mathbb{Q}$-integral polyhedral. 
To check that $f$ is integral polyhedral, we may reduce to the case $\operatorname{dim}(S)=n$. Extend $f$ to a $\mathbb{Q}$-integral polyhedral function on $S$. Let $U$ be a maximal domain of affinity for $f$; since $f$ is $\mathbb{Q}$-integral polyhedral, $U$ is rational polyhedral. Let $\lambda(x)=a_{1} x_{1}+\cdots+a_{n} x_{n}+b$ be the ambient functional for $U$; since $f$ is $\mathbb{Q}$-integral polyhedral, $a_{1}, \ldots, a_{n} \in \mathbb{Z}$ and $b \in \mathbb{Q}$. Write $b=r / s$ in lowest terms. Pick $\left(x_{1}, \ldots, x_{n}\right) \in U \cap \mathbb{Q}^{n}$ such that $s^{\prime} x_{1}, \ldots, s^{\prime} x_{n} \in \mathbb{Z}$ for some integer $s^{\prime}$ coprime to $s$ (such points are dense in $U$ ). Then on one hand $f\left(x_{1}, \ldots, x_{n}\right) \in\left(s^{\prime}\right)^{-1} \mathbb{Z}$, while on the other hand $\lambda(x) \in\left(s^{\prime}\right)^{-1} \mathbb{Z}+b$. We thus have $b \in s^{-1} \mathbb{Z} \cap\left(s^{\prime}\right)^{-1} \mathbb{Z}=\mathbb{Z}$, so $f$ is integral polyhedral.

\section{Tropical Polynomials}

The subject of tropical algebraic geometry has become quite active lately. In that subject, one works not with an ordinary ring but with the tropical semiring, in which the underlying set is $\mathbb{R}$, the "addition" operation is the maximum, and the "multiplication" is ordinary addition. (It is more customary to take the minimum instead of the maximum, for better correspondence with valuation theory, but it is more consistent with the notation in this paper to use the opposite sign convention.)

One is led to ask what a "tropical polynomial" is. If we imagine a polynomial in the variable $x_{1}, \ldots, x_{n}$ to be a "sum" of terms each of which is the "product" of a constant with some of the $x_{i}$ (possibly repeated), we see our answer at once: a tropical polynomial is merely a transintegral polyhedral function in which the slopes have non-negative coefficients. Similarly, a tropical Laurent polynomial, in which we allow "dividing" by $x_{1}, \ldots, x_{n}$ as well, is none other than an arbitrary transintegral polyhedral function.

It is then reasonable to ask for statements identifying tropical (Laurent) polynomials from their restrictions to certain "tropical lines". Here is a sample statement.

Theorem 9.1. Let $f: \mathbb{R}^{2} \rightarrow \mathbb{R}$ be a function. Then the following are equivalent.

(a) The function $f$ is transintegral polyhedral.

(b) The restriction of $f$ to each horizontal and vertical line is transintegral polyhedral.

(c) The restriction of $f$ to each translate of the set

$$
\begin{gathered}
L=\left\{(x, y) \in \mathbb{R}^{2}: x=0, y \leq 0\right\} \cup\left\{(x, y) \in \mathbb{R}^{2}: x \leq 0, y=0\right\} \\
\cup\left\{(x, y) \in \mathbb{R}^{2}: x=y \geq 0\right\}
\end{gathered}
$$

is transintegral polyhedral.

The set $L$ is a typical tropical line; it is the locus where two of the quantities $x, y, 0$ are equal and the third is less. That is, $-x,-y$ could be the valuations of elements $a, b$ of a nonarchimedean ring for which $a+b$ has valuation 0 .

Proof. It is clear that (a) implies (b) and (c). To see that (b) or (c) implies (a), first apply Theorem 6.1 to deduce that $f$ is locally transintegral polyhedral. Then 
apply Lemma 7.3 to each of the four quadrants (in case (b)) or to the closures of each of the three connected components of $\mathbb{R}^{2} \backslash L$ (in case (c)) to deduce that $f$ is transintegral polyhedral.

Many results in tropical algebraic geometry are analogues of statements in ordinary algebraic geometry. For example, one might expect Theorem 9.1 to be the tropical analogue of a statement to the effect that a function of two variables is a polynomial if and only if no matter how we pick one of the variables and a value for that variable, the result is a polynomial function of the other variable. This statement is correct under suitable hypotheses, but not in general. (There is also an analogue for Laurent polynomials, which we leave as an exercise.)

Theorem 9.2. Let $F$ be an infinite field. Let $f: F^{2} \rightarrow F$ be a function for that all $z_{1}, z_{2} \in F$, the restrictions of $f$ to $\left\{z_{1}\right\} \times F$ and $F \times\left\{z_{1}\right\}$ are polynomial functions.

(a) If $F$ is uncountable, then $f$ itself must be a polynomial function.

(b) If $F$ is countable, then $f$ need not be a polynomial function.

We insist that $F$ be infinite so that the evaluation map $F[x] \rightarrow F^{F}$ taking a polynomial to the corresponding function on $F$ is injective. By contrast, if $F$ is finite, then any function from $F$ to itself can be expressed as a polynomial function in infinitely many ways.

Proof. Suppose that for any positive integer $n$, we can find $z_{1,0}, \ldots, z_{0, n} \in F$ such that the functions $f\left(z_{1, i}, \cdot\right): F \rightarrow F$ for $i=0, \ldots, n$ are linearly independent over $F$. Then the function $g: F \rightarrow F$ defined by

$$
g(\cdot)=\operatorname{det}\left(\begin{array}{ccc}
1 & \cdots & 1 \\
z_{1,0} & \cdots & z_{1, n} \\
\vdots & & \vdots \\
z_{1,0}^{n-1} & \cdots & z_{1, n}^{n-1} \\
f\left(z_{1,0}, \cdot\right) & \cdots & f\left(z_{1, n}, \cdot\right)
\end{array}\right)
$$

cannot be the zero function. However, it is a polynomial in its argument, so it has only finitely many roots.

If $z_{2} \in F$ is such that $f\left(\cdot, z_{2}\right)$ is a polynomial of degree at most $n-1$, then $g\left(z_{2}\right)=0$ because we can write the last row of the matrix as a linear combination of the others. By the previous paragraph, there are only finitely many such $z_{2}$. Since this holds for any $n$, there can only be countably many $z_{2}$ such that $f\left(\cdot, z_{2}\right)$ is a polynomial of any degree. Since $F$ is uncountable, this gives a contradiction.

We conclude that the polynomial functions $f\left(z_{1}, \cdot\right)$ span a finite dimensional vector space over $F$. In particular, they all represent polynomials of degree bounded 
by some non-negative integer $n$. Choose $z_{2,0}, \ldots, z_{2, n} \in F$ distinct; for any $z_{1}, z_{2} \in$ $F$, we now have

$$
0=\operatorname{det}\left(\begin{array}{cccc}
1 & \cdots & 1 & 1 \\
z_{2,0} & \cdots & z_{2, n} & z_{2} \\
\vdots & & \vdots & \vdots \\
z_{2,0}^{n} & \cdots & z_{2, n}^{n} & z_{2}^{n} \\
f\left(z_{1}, z_{2,0}\right) & \cdots & f\left(z_{1}, z_{2, n}\right) & f\left(z_{1}, z_{2}\right)
\end{array}\right) .
$$

By expanding in minors along the right column, we express $f\left(z_{1}, z_{2}\right)$ as a polynomial in $z_{2}$ whose coefficients are themselves polynomials in $z_{1}$. This proves (a).

To prove (b), choose an ordering $t_{1}, t_{2}, \ldots$ of the set

$$
T=\left\{\left\{z_{1}\right\} \times F: z_{1} \in F\right\} \cup\left\{F \times\left\{z_{2}\right\}: z_{2} \in F\right\} .
$$

(That is, $T$ is the set of horizontal and vertical lines, not their union.) By induction, we may define functions $f_{n}: t_{1} \cup \cdots \cup t_{n} \rightarrow F$ such that $f_{n}$ restricts to a polynomial of degree $i$ on $t_{i}$ for each $i \in\{1, \ldots, n\}$. These combine to give a function $f: F \times F \rightarrow F$ whose restriction to each $t \in T$ is a polynomial, but these restrictions do not have bounded degree. Hence $f$ cannot itself be a polynomial.

One might also like to view the fact that a function $f: \mathbb{R}^{2} \rightarrow \mathbb{R}$ is locally transintegral polyhedral if and only if the same is true of its restriction to every horizontal line and every vertical line (Theorem 6.1) as the tropical analogue of a statement about polynomials. For this, we might view a locally transintegral polyhedral function as the analogue of something like a Laurent polynomial, but with infinitely many terms. This suggests formulating a statement about entire functions, such as the following.

Theorem 9.3. Let $f: \mathbb{C}^{2} \rightarrow \mathbb{C}$ be a continuous function such that for all $z_{1}, z_{2} \in \mathbb{C}$, the restrictions of $f$ to $\left\{z_{1}\right\} \times \mathbb{C}$ and $\mathbb{C} \times\left\{z_{2}\right\}$ are entire analytic functions. Then $f$ is an entire analytic function.

Proof. Let $C_{i}$ be any circle in the $z_{i}$-plane containing the origin. For any $z_{i}$ in the interior of $C_{i}$, by the Cauchy integral formula applied twice, we have

$$
\int_{C_{1}} \int_{C_{2}} \frac{f\left(w_{1}, w_{2}\right)}{\left(w_{1}-z_{1}\right)\left(w_{2}-z_{2}\right)} d w_{2} d w_{1}=\int_{C_{1}} \frac{f\left(w_{1}, z_{2}\right)}{w_{1}-z_{1}} d w_{1}=f\left(z_{1}, z_{2}\right) .
$$

The left side is infinitely differentiable (the continuity of $f$ makes it valid to differentiate under the integral signs), so $f$ must be as well. Hence $f$ is entire analytic.

Question 9.4. Is there an analogue of Theorem 9.3 in which the restrictions of $f$ are only assumed to be meromorphic? 


\section{Application to $p$-Adic Differential Equations}

We have cited the papers $[3,6]$ for instances of theorems of the sort we have been discussing. To illustrate how these theorems may be applied in practice, we recall just enough of the theory of $p$-adic differential equations to articulate one of these applications.

Definition 10.1. Let $F$ be a field of characteristic zero complete for a nonarchimedean absolute value $|\cdot|$. (We do not require $F$ to be discretely valued; for instance, we might take $F$ to be $\mathbb{C}_{p}$, a completed algebraic closure of the field of $p$-adic numbers.) Let $S \subseteq \mathbb{R}^{n}$ be a bounded transrational polyhedral set. Let $R_{F}(S)$ be the ring whose elements are formal Laurent series

$$
\sum_{i_{1}, \ldots, i_{n} \in \mathbb{Z}} c_{i_{1}, \ldots, i_{n}} t_{1}^{i_{1}} \cdots t_{n}^{i_{n}}, \quad c_{i_{1}, \ldots, i_{n}} \in F
$$

such that for each $r=\left(r_{1}, \ldots, r_{n}\right) \in S$,

$$
\lim _{i_{1}, \ldots, i_{n} \rightarrow \pm \infty}\left|c_{i_{1}, \ldots, i_{n}}\right| e^{-i_{1} r_{1}-\cdots-i_{n} r_{n}}=0 .
$$

(This limit should be interpreted as follows: for any $\epsilon>0$, there are only finitely many $n$-tuples $\left(i_{1}, \ldots, i_{n}\right)$ for which the quantity inside the limit is greater than $\epsilon$.) This ring can be interpreted as the global sections of the structure sheaf of a certain nonarchimedean analytic space, namely the subset of the affine $n$-space with coordinates $t_{1}, \ldots, t_{n}$ defined by the condition

$$
\left(-\log \left|t_{1}\right|, \ldots,-\log \left|t_{n}\right|\right) \in S .
$$

Definition 10.2. Let $\Omega$ be the $R_{F}(S)$-module freely generated by symbols $d t_{1}, \ldots, d t_{n}$. For $j=1, \ldots, n$, define the formal partial derivative $\frac{\partial}{\partial t_{j}}: R_{F}(S) \rightarrow$ $R_{F}(S)$ by the formula

$$
\frac{\partial}{\partial t_{j}}\left(\sum_{i_{1}, \ldots, i_{n} \in \mathbb{Z}} c_{i_{1}, \ldots, i_{n}} t_{1}^{i_{1}} \cdots t_{n}^{i_{n}}\right)=\sum_{i_{1}, \ldots, i_{n} \in \mathbb{Z}} i_{j} t_{j}^{-1} c_{i_{1}, \ldots, i_{n}} t_{1}^{i_{1}} \cdots t_{n}^{i_{n}} .
$$

Define the formal exterior derivative $d: R_{F}(S) \rightarrow \Omega$ by the formula

$$
d(f)=\sum_{j=1}^{n} \frac{\partial}{\partial t_{j}}(f) d t_{j} .
$$

Let $M$ be a finite free $R_{F}(S)$-module. A connection on $M$ is an additive map $\nabla: M \rightarrow M \otimes_{R_{F}(S)} \Omega$ satisfying the Leibniz rule: for $f \in R_{F}(S)$ and $m \in M$,

$$
\nabla(f m)=f \nabla(m)+m \otimes d(f) .
$$

Given a connection $\nabla$, define the maps $D_{1}, \ldots, D_{n}: M \rightarrow M$ by the formula

$$
\nabla(m)=D_{1}(m) \otimes d t_{1}+\cdots+D_{n}(m) \otimes d t_{n} ;
$$

note that $D_{j}$ satisfies the Leibniz rule using the derivation $\frac{\partial}{\partial t_{j}}$ on $R_{F}(S)$. We say that $\nabla$ is integrable if $D_{1}, \ldots, D_{n}$ commute with each other. 
For instance, if $M=R_{F}(S)$, then $\nabla=d$ is an integrable connection. We next give a numerical measure of the failure of a given integrable connection to have this special form.

Definition 10.3. For $r \in S$, define the norm $|\cdot|_{r}$ on $R_{F}(S)$ by the formula

$$
\left|\sum_{i_{1}, \ldots, i_{n} \in \mathbb{Z}} c_{i_{1}, \ldots, i_{n}} t_{1}^{i_{1}} \cdots t_{n}^{i_{n}}\right|_{r}=\sup \left\{\left|c_{i_{1}, \ldots, i_{n}}\right| e^{-i_{1} r_{1}-\cdots-i_{n} r_{n}}: i_{1}, \ldots, i_{n} \in \mathbb{Z}\right\} ;
$$

the definition of $R_{F}(S)$ makes this quantity finite, and in fact ensures that the supremum is achieved for at least one tuple $\left(i_{1}, \ldots, i_{n}\right) \in \mathbb{Z}^{n}$. Let $E_{r}$ be the completion of Frac $R_{F}(S)$ for this norm; then each $\frac{\partial}{\partial t_{j}}$ extends continuously to a map $\frac{\partial}{\partial t_{j}}: E_{r} \rightarrow E_{r}$, while $d$ extends continuously to a map $d: E_{r} \rightarrow \Omega \otimes_{R_{F}(S)} E_{r}$.

Definition 10.4. Let $M$ be a finite free $R_{F}(S)$-module equipped with an integrable connection $\nabla$. For $r \in S$, we extend $\nabla$ to a map $\nabla: M \otimes_{R_{F}(S)} E_{r} \rightarrow M \otimes_{R_{F}(S)}$ $\Omega \otimes_{R_{F}(S)} E_{r}$; we correspondingly extend $D_{1}, \ldots, D_{n}$ to maps from $M \otimes_{R_{F}(S)} E_{r}$ to itself. Let $N$ be a subquotient of $M \otimes_{R_{F}(S)} E_{r}$ in the category of $E_{r}$-modules on which $D_{1}, \ldots, D_{n}$ act. Pick a basis of $N$, and use it to define a supremum norm on $N$ compatible with the norm $|\cdot|_{r}$ on $E_{r}$. Let $\left|D_{j}\right|_{\text {spect, } N}$ denote the spectral norm of $D_{j}$ on $N$, that is,

$$
\left|D_{j}\right|_{\text {spect }, N}=\limsup _{s \rightarrow \infty}\left|D_{j}^{s}\right|_{N}^{1 / s}
$$

where $\left|D_{j}^{s}\right|_{N}$ denotes the operator norm of $D_{j}^{s}$ on $N$ for the chosen norm. This quantity does not depend on the choice of the norm. Define the intrinsic generic radius of convergence of $N$ to be

$$
\min \left\{\frac{\left|D_{j}\right|_{\text {spect }, E_{r}}}{\left|D_{j}\right|_{\text {spect }, N}}: j \in\{1, \ldots, n\}\right\} .
$$

(This indeed has something to do with the radius of convergence of certain horizontal sections of a certain differential module. See [6, Proposition 1.2.14] for a bit more explanation, and $[4, \S 9.7]$ for much more discussion.)

Let $N_{1}, \ldots, N_{h}$ be the Jordan-Hölder constituents of $M \otimes_{R_{F}(S)} E_{r}$, i.e. the successive quotients in a maximal filtration by $E_{r}$-submodules preserved by $D_{1}, \ldots, D_{n}$. Define the multiset of subsidiary radii of $M$ at $r$ to consist of, for $j=1, \ldots, h$, the intrinsic generic radius of convergence of $N_{j}$ with multiplicity $\operatorname{dim}_{E_{r}}\left(N_{j}\right)$.

Remark 10.5. In the case of residual characteristic zero, the subsidiary radii are related to the classical notion of irregularity for a meromorphic connection on a complex analytic variety. See [5] for discussion of that case. In the case of residual characteristic $p>0$, the subsidiary radii are related to wild ramification of maps between varieties over a field of positive characteristic; see [2]. 
We can now state part of [6, Theorem 3.3.9], which governs the variation of the subsidiary radii, and then explain how a polyhedrality theorem intervenes in the proof.

Theorem 10.6. Let $M$ be a finite free $R_{F}(S)$-module of rank $m$, equipped with an integrable connection $\nabla$. For $r \in S$, let $f_{1}(M, r) \geq \cdots \geq f_{m}(M, r)$ be the non-negative real numbers such that the subsidiary radii of $M$ at $r$ are equal to $e^{-f_{1}(M, r)}, \ldots, e^{-f_{m}(M, r)}$. Define $F_{i}(M, r)=f_{1}(M, r)+\cdots+f_{i}(M, r)$. Then for $i=1, \ldots, m$, the function $r \mapsto m ! F_{i}(M, r)$ is transintegral polyhedral; for $i=m$, the function $r \mapsto F_{m}(M, r)$ is also transintegral polyhedral.

It turns out to be difficult to analyze the $F_{i}(M, r)$ on all of $S$ directly. Instead, the proof goes by checking transintegral polyhedrality on each transrational line, then invoking Theorem 7.4. The point is that by a change of coordinates (replacing $t_{1}, \ldots, t_{n}$ by certain monomials) we can reduce the study of any transrational line to the study of a line parallel to one of the coordinate axes, say the first one. In that case, there is no harm in burying $t_{2}, \ldots, t_{n}$ in the base field (i.e. replacing $F$ by the completion of the rational function field $F\left(t_{2}, \ldots, t_{n}\right)$ for an appropriate Gauss norm) as long as we keep track of the derivations with respect to $t_{2}, \ldots, t_{n}$. Although the remaining calculation is still quite intricate, it would have been immeasurably more so without the reduction to the one-dimensional case.

\section{Other Questions}

We conclude by mentioning a question for which our ignorance of the answer remains somewhat frustrating. This should illustrate the extent to which the restriction to integral slopes is crucial for the arguments of this paper.

Question 11.1. Let $f: \mathbb{R}^{2} \rightarrow \mathbb{R}$ be a function whose restriction to each line is polyhedral. Is $f$ necessarily polyhedral? The example $f(x, y)=x y$ shows that it is insufficient to require the restriction to each line parallel to one of the coordinate axes to be polyhedral.

\section{Acknowledgments}

Thanks to David Speyer for helpful discussions. The first author was supported by NSF CAREER grant DMS-0545904 and the NEC Research Support Fund. The second author was supported by the Paul E. Gray Fund of MIT's Undergraduate Research Opportunities Program (UROP).

\section{References}

1. N. Bourbaki, Fonctions d'une Variable Réelle (Hermann, 1958).

2. K. S. Kedlaya, Swan conductors for $p$-adic differential modules, I: A local construction, Alg. Number Th. 1 (2007) 269-300. 
3. K. S. Kedlaya, Semistable reduction for overconvergent F-isocrystals, III: Local semistable reduction at monomial valuations, Compos. Math. 145 (2009) 143-172.

4. K. S. Kedlaya, $p$-adic differential equations (version of 19 Jan 2009), preprint at http:// math.mit.edu $/{ }^{\sim}$ kedlaya/papers/.

5. K. S. Kedlaya, Good formal structures for flat meromorphic connections, I: Surfaces, arXiv:0811.0190v3.

6. K. S. Kedlaya and L. Xiao, Differential modules on $p$-adic polyannuli, arXiv:0804.1495v4, to appear in J. Institut Math. Jussieu.

7. R. T. Rockafellar, Convex Analysis (Princeton Univ. Press, 1970).

8. D. F. Young, When does unique local support ensure convexity?, Trans. Amer. Math. Soc. 347 (1995) 1323-1329. 